\title{
Análisis exploratorio del campo de fuerzas alrededor de una cultura de simplicidad en las empresas del sector petroquímico de Cartagena de Indias
}

\author{
Exploratory analysis of the field of forces around a culture of simplicity in the companies of the \\ petrochemical sector of Cartagena de Indias
}

DOI: 10.21803/adgnosis.v8i8.363

\section{Resumen}

La complejidad es inherente a la naturaleza de las organizaciones por ello requiere ser comprendida, gestionada a través de la construcción de espacios semánticos y formas de acción tanto de individuos como de colectivos. En forma paradójica, un elemento de la complejidad es la simplicidad. De ahí que explorar la simplicidad en las organizaciones es un componente clave para canalizar la complejidad y un desafío del Management, en especial del Campo de la Estrategia. La simplicidad es por tanto un modo particular de comprender la realidad. Es también una capacidad cognitiva que debiera desarrollar el personal de la organización. La Simplicidad es la paradoja misma de la complejidad en la que "ve lo uno, y ve lo múltiple, pero no puede ver que lo uno puede, al mismo tiempo ser múltiple" (Balmes,1860).

Palabras clave: Simplicidad, Complejidad, Cultura Organizacional, Campo de Fuerzas

\section{Abstract}

Complexity is inherent to the nature of organizations and therefore requires to be understood, managed through construction of semantic spaces and forms of action of both individuals and human collectives. Paradoxically, one element of complexity is simplicity. Hence, exploring simplicity in organizations is a key component for channeling complexity and a management challenge, especially in the Field of Strategy. Simplicity is therefore a particular way of understanding reality. It is also a cognitive capacity that should be developed by the organization staff. Simplicity is the very paradox of complexity in which "you see the one, and see the multiple, but you can not see that one can be simultaneously one and be multiple" (Balmes, 1860).

Keywords: Simplicity, Complexity, Organizational Culture, Field of Forces

\author{
Juan Carlos Robledo Fernandez ${ }^{1}$ \\ jrobledo1962@gmail.com \\ Gabriel Ruiz Andrade ${ }^{2}$ \\ gabruiz@uabc.edu.mx \\ Jorge Mario Rodas Cadavid ${ }^{\star}$ \\ William Enrique Meriño Molina*
}

\section{Cómo citar este artículo:}

Robledo J., Ruiz G., Rodas J. \& Meriño W. (2019). Análisis exploratorio del campo de fuerzas alrededor de una cultura de simplicidad en las empresas del sector petroquímico de Cartagena de Indias 8(8), p. 99-115 DOI: 10.21803/ adgnosis.v7i7.296

1 Director Programa Maestría en Administración y de la Línea de Investigación en Estrategia y Gestión Adscrita al Instituto de Estudios para el Desarrollo IDE, de la Facultad de Economía y Negocios de la Universidad Tecnológica de Bolívar, PhD. en administración. Magister en administración. Economista. ORCID https://orcid.org/0000-0001-6303-8688 


\section{Introducción}

La inherente naturaleza compleja de las organizaciones es suficientemente atractiva para invitar a los investigadores a interesarse por estudiarla. En este sentido, una de las aproximaciones teóricas de análisis es comprender la complejidad a través de la simplicidad vista como un aspecto de la cultura organizacional. Por ello, el objetivo principal del estudio exploratorio que se presenta en este documento, ha sido identificar el campo de fuerzas alrededor de una cultura de la simplicidad en una primera aproximación. Para ello, se ha realizado una investigación rigurosa que ha tenido como unidad de análisis dos empresas del sector petroquímico de la ciudad de Cartagena de Indias.

El estudio aborda la temática de la Simplicidad como un componente que encaja dentro del marco de la cultura organizacional, la idea fue buscar factores que nos indiquen como desde la organización podemos incorporar elementos de simplicidad para hacer la vida cotidiana organizacional menos compleja. En este sentido, se propusieron teóricamente cinco categorías: La comodidad, la ausencia de confusión, la conciencia de su trabajo, las habilidades de síntesis y el espíritu de ayuda. A cada categoría se le asignaron cinco ítems (o factores), que se llevaron a una prueba empírica a través de un instrumento contestado por 119 trabajadores de dos empresas grandes del sector petroquímico de Cartagena.

Metodológicamente la Investigación usa el marco analítico del modelo factorial de primeros componentes con un análisis confirmatorio por rotación Varimax, el cual superó las pruebas estadísticas de las hipótesis requeridas, lo que permitió obtener una matriz de componentes rotados que agrupa satisfactoriamente los factores, emergiendo seis categorías empíricas de aproximación a la simplicidad que explican en más del 66\% la varianza, estas son en su orden: Conciencia de su trabajo, Competencias específicas para el trabajo, Capacitación, Habilidad de síntesis e innovación, Autonomía y Bienestar, y Libertad de acción.

Se llega a la propuesta del modelo conceptual del campo de fuerzas en el que se pone en contraste la propuesta teórica y los resultados empíricos, además de establecer en las conclusiones siete hallazgos empíricos en la línea de aproximación a la comprensión de la complejidad como fenómeno organizacional a través de la gestión de la simplicidad.

El estudio exploratorio no pretende hacer generalizaciones universales, sin embargo, introduce lo que se denomina "generalización analítica" con base en el estudio empírico de dos casos de empresas del sector petroquímico de la ciudad de Cartagena de Indias.

Finalmente, se anexan el modelo de instrumento que obtuvo una validación de Cronbach de 0,92, y las matrices de extracción sin rotación y con rotación, además del anexo de pruebas de hipótesis.

\section{Revisión de literatura}

Para hacer referencia a la simplicidad en el presente estudio exploratorio, resulta apropiado en primer lugar comprender que lo que se propone es explorar qué factores del orden organizacional puede permitir lidiar con la complejidad específicamente en las organizaciones y particularmente desde la consolidación de una cultura organizacional. Por tanto, en esta Investigación la complejidad se asume como una paradoja de la simplicidad, un oxímoron, al respecto Ashkenas (2007) expresó:

Large organizations are by nature complex, but over the years circumstances have conspired to add layer upon layer of complexity to how businesses are structured and managed" "Complexity is the cumulative by-product of organizational changes, big and small, that over the years weave complications (often invisibly) into the ways that work is done.

Se desprende de aquí, que la complejidad es inherente a la naturaleza de las organizaciones y por ende requiere ser comprendida, gestionada a través del diseño, y la creación de "formas de ser" que gene- 
ralmente se desarrollan en los "espacios semánticos de la cultura organizacional”. En esta ocasión, este espacio semántico y de acción se propone sea denominado "cultura de simplicidad". Es por ello que se ha escogido el sector petroquímico como una primera aproximación al fenómeno de simplicidad haciendo la salvedad que este término no hace referencia al "simplismo o a las acciones simplistas". En esta consideración Morin (1994) plantea respecto de la complejidad y la empresa "tanto la simplicidad relacionada con el orden como la complejidad relacionada con el desorden, son necesarias para la existencia de las organizaciones en la medida que las organizaciones tienen necesidad de orden y desorden” (p.126). En este sentido se le atribuye al orden el carácter simplificador, y paradójicamente es la búsqueda de este orden lo que genera complejidad (Robledo \& Ruiz, 2017, p.19).

Por tanto, es menester para los tomadores de decisiones organizacionales, de quienes las diseñan y les crean su arquitectura, considerar el mandamiento de reducir la complejidad conocida, considerando lo que plantea la navaja de Occam (citado en Nisbett, 2016) en su libro Mindware. Las teorías deben ser concisas hay que afeitar de ellas los conceptos innecesarios. Hay que "afeitar" de las organizaciones las propuestas de hacer de las cosas lo más difíciles porque así son o porque así las han diseñado. Es por ello relevante empezar investigaciones exploratorias en este campo de la simplicidad en las organizaciones, atendiendo el criterio de que si en el ámbito científico gana la teoría más simple capaz de explicar los hechos, entonces en el ámbito de una empresa u organización, gana ventaja aquella que está preparada desde su cultura organizacional para actuar desde una cultura de la simplicidad, entendiéndola como una actitud individual y colectiva apropiada para enfrentar la multitud de las cosas sin caer en el exagerado reduccionismo.

Ahora bien, entendemos esta actitud individual y colectiva desde la premisa "lo que es sensato en la ciencia también puede ser sensato en los negocios”. Abordar entonces la simplicidad como objeto de estudio en las organizaciones y éstas como unidades de análisis, implica de alguna forma poder operar el principio de parsimonia el cual obliga a que los fenómenos se expliquen primero en el nivel más simple posible y poco a poco añadir complicaciones en la medida que sea solo necesario. Atendiendo tal como lo plantea Nisbett (2016) “el esfuerzo por explicar las cosas en un nivel más bajo de la jerarquía puede ser útil incluso si la conclusión última es que hay propiedades emergentes que no permiten una explicación completa en términos de procesos subyacentes más simples" (p. 308). En otras palabras, la complejidad también existe de forma autónoma por sí misma en distintas categorías (o dimensiones) del mundo físico y social, y en este último los seres humanos aumentamos la complejidad en el entramado de las relaciones sociales que se construyen en su vida cotidiana como respuesta a las dinámicas propias de su entorno.

De otra parte, el ser humano en la complejidad del mundo material y social que construye, está siempre buscando principios de simplificación y mecanismos para aplicarlos asumiendo el costo reduccionista de la comprensión de un fenómeno en concreto. Sin embargo, tal como lo manifiestan Di Tore et al. (2014) a nivel de las organizaciones es necesario tener claro a que se refiere la simplicidad como estrategia y como medio para actuar y servir a los propósitos empresariales y de la propia organización. Por tanto, los principios de simplicidad se ven incorporados en los aspectos perceptivos que incluye el mundo de las cosas en su entorno, la realidad simbólica y las señales emitidas por cada sujeto y objeto en su respectivo campo semántico de acción, lo que en sí misma también es parte de la complejidad circundante de la organización y su propia realidad.

De aquí que, retomando a Morin (1994) cuando dice "la voluntad de imponer al interior de una organización un orden implacable no es suficiente" (p.126). Se refiere a la simplicidad como un elemento coordinador entre el orden y el desorden, pero partiendo del acto voluntario de cada miembro de la organización. Por tanto, en esta investigación se acoge la definición de simplicidad como la ausencia de confusión con lo que cada miembro de la organización se 
siente cómodo a la hora de desarrollar sus tareas, a cualquier nivel en el que se desenvuelva, como lo manifiesta Segall (2012) la simplicidad es aquello con lo que los seres humanos se sienten instintivamente cómodo. Esto es equivalente a claridad, evidencia y ausencia de confusión. Se trata de una cosa que es fácil recordar y difícil olvidar. En este sentido la simplicidad es un modo de comprender la realidad en la que los individuos desarrollan hacia ella una actitud específica y disminuyendo la posibilidad de confusión. Además, se agrega la conciencia que trabajador tenga de su trabajo, las condiciones de comodidad para desarrollarlo, las habilidades de síntesis para abordar problemas y su espíritu de ayuda para con los compañeros de trabajo.

Ahora bien, al concebirse la simplicidad como un modo de comprender la realidad, ésta, se traduce en sí misma en un modo de conocer el mundo cotidiano, lo que advierte la existencia de un propósito cognitivo. Esto hace que conectar la simplicidad bajo un modelo de gestión que permita hacer el buen gobierno de las personas al interior de las organizaciones escape del modelo tradicional de la administración basado en una racionalidad cartesiana que busca la optimización, minimización y maximización.

De otra parte, toma relevancia para este estudio, lo que expresa Hoffman (1987) la idea central es la integración de afecto y cognición más allá del procesamiento de la información. En otras palabras, se busca una integración con capacidad comprensiva del fenómeno organizacional tanto en sus funciones como en sus derroteros estratégicos por la sostenibilidad. En este sentido y complementando la definición anterior de simplicidad, ésta también se comprende para la organización como la capacidad y habilidad de las personas para trabajar bajo estados plenos de conciencia, desarrollando habilidades de precisión, síntesis, ingenio, creatividad, método y espíritu de ayuda. Se aclara nuevamente aquí, que no puede confundirse simplicidad con simplismo. Este último, es una actitud humana basada en elementos de ignorancia, facilismo, negligencia y una total desatención por un comportamiento prosocial.
En el sentido de lo descrito en el párrafo anterior, es apropiado acoger lo expuesto por Katsuhiko (1987) al expresar cuando se intenta construir un modelo, debe establecerse un equilibrio entre la simplicidad del modelo y la exactitud de los resultados del análisis. Lo que nos lleva a contemplar lo expresado por De Bono (1998) la búsqueda de la simplicidad nos ha de permitir volver a pensar en todo, no solo en las áreas problemática. De esta forma, la simplicidad también se asume como un medio apropiado para abordar los problemas de la complejidad en un mundo no solo caótico sino también desordenado. En este sentido la fuerza profunda de la simplicidad la resumió Sand (2010) cuando expresó la simplicidad es lo más difícil de conseguir en este mundo, es el último límite de la experiencia y el último esfuerzo del genio.

De otro lado, la simplicidad desde un enfoque filosófico advierte sus propias contradicciones, tal como lo manifestó Balmes (1860) "la simplicidad ve lo uno y ve lo múltiple, pero no puede ver que lo uno puede, al mismo tiempo ser múltiple” (p.26). Lo paradójico en esta observación filosófica, es que la simplicidad en sí misma es compleja. Puede separar lo que está ligado o puede bien unificar lo que está disperso y diverso. Todo esto es posible porque el hombre es un ser biológico, pero al mismo tiempo es un ser evidentemente cultural, meta- biológico, que vive en mundo construido desde el lenguaje donde se constituye su humanidad y su ontogenia con sus ideas y su propia conciencia (Robledo y Santamaría, 2016, p. 6).

La simplicidad, es entonces, una manifestación de las creencias mismas de cada ser humano la cual desarrolla y comparte dentro y fuera de las organizaciones además de constituir una forma de conciencia colectiva de que existen partes no percibidas, como la expresara Balmes (1860) "Aquí no puede menos que observar como el análisis trascendental confunden a los que no admiten la simplicidad en los seres pensantes; pues encontramos que la simplicidad es primero que la composición y que ésta no puede concebirse, sino presupone aquella"( p.29). La simplicidad es por tanto una ley natural de todo ser, en él habita, y por tanto es posible conjugarla en lo individual 
y lo colectivo al interior de las organizaciones.

Finalmente, la simplicidad incorporada a los modelos de gestión, puede actuar como una capacidad distintiva de muy difícil imitación toda vez que corresponde al comportamiento humano dentro de la organización que se refleja y actúa probablemente desde una cultura organizacional. Es, sin embargo, pertinente establecer que, desde el aspecto de la estrategia, la simplicidad se vincula al desarrollo del conocimiento organizacional y se vincula a éste en perspectivas distintas tanto desde lo tácito como lo explícito. En este punto, este estudio exploratorio acoge la hipótesis de trabajo creada por Robledo et al. (2015) en su libro de Gestión del Conocimiento Organizacional, cuando expresa "Toda acción, todo comportamiento y en particular todo discurso alrededor de la creación de conocimiento organizacional reposa sobre los modelos mentales de la realidad específica socialmente construida por cada individuo dentro de cada organización” (p. 71).

El conocimiento le puede aportar al estudio de la complejidad en la organización a través de la simplicidad, mayor capacidad de respuesta al entorno, en este sentido Grant (1996) en su teoría de la firma basada en el conocimiento logró establecer que toda organización cumple con una función integradora de conocimiento, que éste a pesar de ser complejo se integra desde las rutinas organizacionales que se conectan con la base de la cultura organizacional. Al igual, Wernerfelt (1984) logró establecer que a pesar de que se asumía la homogeneidad de los recursos dentro de las firmas, había una diferencia y está en los recursos intangibles que otorgan capacidades adicionales que permiten la diferencia, estos recursos son tanto complejos como simples, pero igual constituyentes de rutinas organizacionales. De otro lado la simplicidad solo podría actuar como parte de un modelo de gestión si está mediada por el aprendizaje organizacional para la creación de capacidades distintivas denominadas por Teece et al. (1997) Capacidades Dinámicas, en su teoría de la firma basada en los recursos y capacidades dinámicas.

La Simplicidad es también un asunto de estrate- gia, se puede decir que este es uno de los más grandes desafíos del Management para la comprensión de la complejidad organizacional. Puede también expresarse este desafío tal como lo manifestó el profesor Lewin (1951) la mejor manera de comprender, entender y explicar algo, es intentar cambiarlo. Esto puede también expresarse desde el autoconocimiento, y la capacidad de comprender el entorno, tal como lo manifiesta el profesor Gore (2012) los humanos vivimos en organizaciones tal como los peces viven en el agua. Sólo que a los peces les resulta difícil entender que están húmedos, y a nosotros nos resulta en ocasiones difícil entender y comprender a las organizaciones y su complejidad.

En consecuencia, abordar la exploración de un campo de fuerzas delineado desde la simplicidad como instrumento cognitivo para comprender la acción dentro de la organización y sus efectos externos, compromete no solo el entendimiento de la organización misma, sino también facilita explicar los procesos de la comunicación organizacional y el aprendizaje permanente, a lo que en su momento Argyris (1997) llamo los ajustes ecológicos en la organización. Estos ajustes permiten inferir que las organizaciones no tienen realmente objetivos sino muchas restricciones por superar (Charles, 1986). Las restricciones son en su medida un marco generador de complejidad organizacional.

Precisamente son las restricciones las que detonan de cierta forma el marco complejo de toda organización al imponer límites o barreras a los tomadores de decisiones, y se advierte entonces el reconocimiento de que toda organización funciona como un sistema social humano que requiere desarrollar la capacidad de "conciencia organizacional”, comprendida esta como la capacidad de auto reconocimiento de su propia complejidad, que se ve a sí misma como un "ser organizacional” y no como un sistema mecánico de tipo lineal que sirve solo a propósitos de transacción. Al respecto de esta conciencia organizacional, Tomasko (1993) afirma que la simplicidad en el lugar de trabajo es una virtud fácilmente transferible. No exige una cultura empresarial arraigada en la cultura Zen. No obstante, si requiere un esfuerzo 
consciente de eliminación del trabajo innecesario". Se desprende de aquí la idea de que todo empleado en teoría debería desarrollar una "conciencia de su trabajo en la organización, y ésta debe corresponder a la conciencia misma de la organización.

Para efectos de exploración, se les han asignado las siguientes categorías teóricas para orientar el análisis en el presente estudio:

1. La comodidad, se define como las condiciones de tipo físico e intangible apropiadas, que cada trabajador requiere tener como mínimo para desempeñarse apropiadamente en su lugar de trabajo.

\section{La Ausencia de Confusión en el trabajo,} son las acciones que la empresa ejerce para que cada trabajador tenga pleno conocimiento sobre el desempeño de sus funciones y sus procesos de aprendizaje.

3. Conciencia de su trabajo, es aquella posición cognitiva por parte del trabajador en lo que respecta al conocimiento mismo de su trabajo y su relación con otras áreas de la empresa.

4. Habilidades de Síntesis, se refiere a la capacidad que tiene cada trabajador para abstraer problemas, buscar soluciones, además de preparar informes concretos y precisos.

5. Espiritu de Ayuda, es la condición propia de cada trabajador para juntarse con otras personas y apropiar trabajos en equipo, cooperar, colaborar con otros en el logro de sus objetivos y el de los demás.

\section{Metodología}

Para el presente estudio exploratorio se configuró una muestra de 119 trabajadores del sector petroquímico de la ciudad de Cartagena de Indias correspondiente a una empresa estatal y una privada, demográficamente constituida el $75 \%$ por el género masculino y el $25 \%$ por género femenino, empleados en las áreas de ingeniería, operaciones, investigación y desarrollo, analistas de laboratorio y personal administrativo. La edad de los informantes oscila entre los 22 y los 60 años, con una antigüedad media en el trabajo de 9,25 años, en la que los informantes presentan en un $67 \%$ formación de posgrados, ocupando mandos medios y altos en un $26 \%$ de las veces.

Para la recolección de los datos se diseñó un instrumento estructurado en escala de Likert con valoración de 1 a 5 , en donde el 1 equivale a total desacuerdo y el 5 a total acuerdo. El instrumento se estructuró con 25 ítems dividido en cinco categorías propuestas de 5 ítems cada una. Las categorías son:1). Comodidad (CO), 2). Ausencia de confusión en el trabajo (AC), 3). Conciencia de su trabajo (CT), 4). Habilidades de Síntesis (HS), 5). Espiritu de ayuda (EA). La aplicación del instrumento se hizo persona a persona durante un tiempo de 3 meses, se aplicó un protocolo en el que se entregó a cada participante una hoja de consentimiento informado. El instrumento (ver anexo 1) se aplicó en los lugares de trabajo y se les prometió a los informantes la confidencialidad de sus nombres, cada una de los informantes participó de manera voluntaria y consentida.

El instrumento fue validado a través de una prueba piloto con la participación inicial de 40 informantes y se verifico su consistencia y fiabilidad interna obteniendo un Alpha de Cronbach de 0,92 (Cronbach, 1951) (ver anexo 4). Una vez verificada la consistencia se procedió a su ejecución hasta completar el tamaño de la muestra configurada. Por ser un estudio exploratorio no se usó una muestra probabilística sino de configuración.

Los ítems asociados a cada categoría teórica inicial son el producto de las entrevistas realizadas con trabajadores previo al diseño del instrumento. Se hacen entrevistas semiestructuradas con el propósito de rescatar aquellas características que pueden ser constituidos como ítem para incorporar al instrumento. Los ítems asociados a las categorías son entonces incorporados para verificación de consistencia a través del Alpha de Cronbach, y posteriormente con la técnica de análisis factorial para verificar la 
agrupación. Estas categorías usadas se consideran de tipo de dirección ya que cada ítem establecido busca evaluar de cómo es tratado el asunto por cada informante, si favorable o desfavorablemente (Krippendorff, 1990, p. 102).

Dado que se propuso un estudio exploratorio, se asume como técnica el análisis factorial de primeros componentes (o llamado también de componentes principales) con extracción sin rotación y extracción confirmatoria por método Varimax, en el entendido de que se buscaba descubrir los primeros componentes que agrupan al conjunto de características asociadas a las categorías observadas empíricamente y que puedan estar explicando al menos el $60 \%$ del constructo en estudio en este caso la simplicidad. Para el tratamiento de los datos se usó el Software SPSS versión 23 en español.
En cuanto al modelo factorial se realizaron las pruebas pertinentes con relación al KMO (Kaiser-Meyer-Olkin) que arrojó un resultado de 0,856 considerado muy bueno para proseguir con el uso de la técnica factorial además se complementa esta prueba al obtener una significancia frente a la prueba de esfericidad de Bartlett menor al $5 \%$, lo que confirmó que el método de extracción era factible para los datos recolectados y valorados en una matriz (Ver anexo 4).

\section{Resultados}

Para la extracción primaria sin rotación se usa el criterio de las comunidades mayores a 1 permitiendo obtener el porcentaje de varianza total explicada en la que se obtienen seis (6) componentes que explican en un poco más del $66,5 \%$ la varianza, tal como se muestra en la siguiente tabla 1.

Tabla 1.

Porcentaje de la varianza explicada sin rotación

\begin{tabular}{|c|c|c|c|c|c|c|}
\hline \multirow[b]{2}{*}{ Componente } & \multicolumn{3}{|c|}{ Autovalores iniciales } & \multicolumn{3}{|c|}{ Sumas de extracción de cargas al cuadrado } \\
\hline & Total & \% de varianza & $\%$ acumulado & Total & \% de varianza & \% acumulado \\
\hline 1 & 9,178 & 36,710 & 36,710 & 9,178 & 36,710 & 36,710 \\
\hline 2 & 2,124 & 8,495 & 45,206 & 2,124 & 8,495 & 45,206 \\
\hline 3 & 1,799 & 7,195 & 52,401 & 1,799 & 7,195 & 52,401 \\
\hline 4 & 1,402 & 5,607 & 58,008 & 1,402 & 5,607 & 58,008 \\
\hline 5 & 1,086 & 4,344 & 62,352 & 1,086 & 4,344 & 62,352 \\
\hline 6 & 1,027 & 4,109 & 66,460 & 1,027 & 4,109 & 66,460 \\
\hline 7 & 924 & 3,696 & 70,157 & & & \\
\hline 8 & 894 & 3,578 & 73,734 & & & \\
\hline 9 &, 759 & 3,037 & 76,771 & & & \\
\hline 10 &, 722 & 2,889 & 79,660 & & & \\
\hline 11 & ,665 & 2,662 & 82,322 & & & \\
\hline 12 & .543 & 2,171 & 84,492 & & & \\
\hline 13 &, 535 & 2,141 & 86,633 & & & \\
\hline 14 & .463 & 1,851 & 88,484 & & & \\
\hline 15 &, 447 & 1,787 & 90,271 & & & \\
\hline 16 & ,389 & 1,558 & 91,828 & & & \\
\hline 17 & 340 & 1,361 & 93,189 & & & \\
\hline 18 & 309 & 1,237 & 94,426 & & & \\
\hline 19 & 263 & 1,053 & 95,479 & & & \\
\hline 20 & 255 & 1,022 & 96,501 & & & \\
\hline 21 & ,220 & ,881 & 97,382 & & & \\
\hline 22 & ,206 & ,823 & 98,205 & & & \\
\hline 23 & 178 & ,711 & 98,916 & & & \\
\hline 24 & 153 &, 610 & 99,526 & & & \\
\hline 25 &, 118 & .474 & 100,000 & & & \\
\hline
\end{tabular}

Fuente: Elaboración propia. 
La extracción sin rotación entrega seis primeros componentes que cumplen con los criterios de reducción de la técnica y que por saturación de cada ítem o variable se obtienen seis factores en orden de varianza explicada. Luego se aplica un análisis confirmatorio por rotación varimax de la solución original, la cual se realiza con el objetivo de mejorar la interpretación de la estructura factorial. La rotación Varimax, es un método de rotación ortogonal, lo que quiere decir que respeta la independencia entre factores de la solución inicial. Este método minimiza el número de variables que tienen saturaciones altas de cada factor. En la tabla 2 se presenta la matriz de varianza explicada con rotación Varimax.
A partir de la acción confirmatoria por uso del método de Rotación Varimax, se puede observar que la agrupación de factores se hace más homogénea y satura de manera apropiada la extracción brindando una mayor oportunidad de categorización y análisis de los resultados obtenidos. La rotación confirma los seis componentes identificados en la primera extracción sin rotación, el nivel de explicación de la varianza se mantiene relativamente igual, lo que está indicando estabilidad. Las pruebas de esfericidad y KMO se cumplen sin ningún problema (Ver anexo 4).

A continuación, en la tabla 2, se presenta la matriz de extracción bajo la condición confirmatoria y la extracción rotada bajo el método Varimax.

Tabla 2.

Explicación de varianza con rotación Varimax

\begin{tabular}{|c|c|c|c|c|c|c|c|c|c|}
\hline \multirow[b]{2}{*}{ Componente } & \multicolumn{3}{|c|}{ Autovalores iniciales } & \multicolumn{3}{|c|}{ Sumas de extracción de cargas al cuadrado } & \multicolumn{3}{|c|}{ Sumas de rotación de cargas al cuadrado } \\
\hline & Total & \% de varianza & \% acumulado & Total & \% de varianza & \% acumulado & Total & \% de varianza & \% acumulado \\
\hline 1 & 9,178 & 36,710 & 36,710 & 9,178 & 36,710 & 36,710 & 3,946 & 15,784 & 15,784 \\
\hline 2 & 2,124 & 8,495 & 45,206 & 2,124 & 8,495 & 45,206 & 3,627 & 14,506 & 30,290 \\
\hline 3 & 1,799 & 7,195 & 52,401 & 1,799 & 7,195 & 52,401 & 3,157 & 12,627 & 42,917 \\
\hline 4 & 1,402 & 5,607 & 58,008 & 1,402 & 5,607 & 58,008 & 2,268 & 9,071 & 51,987 \\
\hline 5 & 1,086 & 4,344 & 62,352 & 1,086 & 4,344 & 62,352 & 2,047 & 8,190 & 60,177 \\
\hline 6 & 1,027 & 4,109 & 66,460 & 1,027 & 4,109 & 66,460 & 1,571 & 6,283 & 66,460 \\
\hline 7 &, 924 & 3,696 & 70,157 & & & & & & \\
\hline 8 &, 894 & 3,578 & 73,734 & & & & & & \\
\hline 9 &, 759 & 3,037 & 76,771 & & & & & & \\
\hline 10 &, 722 & 2,889 & 79,660 & & & & & & \\
\hline 11 &, 665 & 2,662 & 82,322 & & & & & & \\
\hline 12 &, 543 & 2,171 & 84,492 & & & & & & \\
\hline 13 &, 535 & 2,141 & 86,633 & & & & & & \\
\hline 14 &, 463 & 1,851 & 88,484 & & & & & & \\
\hline 15 &, 447 & 1,787 & 90,271 & & & & & & \\
\hline 16 &, 389 & 1,558 & 91,828 & & & & & & \\
\hline 17 &, 340 & 1,361 & 93,189 & & & & & & \\
\hline 18 &, 309 & 1,237 & 94,426 & & & & & & \\
\hline 19 &, 263 & 1,053 & 95,479 & & & & & & \\
\hline 20 &, 255 & 1,022 & 96,501 & & & & & & \\
\hline 21 &, 220 &, 881 & 97,382 & & & & & & \\
\hline 22 &, 206 &, 823 & 98,205 & & & & & & \\
\hline 23 & ,178 & ,711 & 98,916 & & & & & & \\
\hline 24 & 153 &, 610 & 99,526 & & & & & & \\
\hline 25 &, 118 &, 474 & 100,000 & & & & & & \\
\hline
\end{tabular}

Método de extracción: análisis de componentes principales.

Fuente: Cálculos efectuados por los autores con el SPSS. 
Las condiciones de independencia se mantienen, y se confirma por rotación que son seis los componentes que explican un poco más del $66 \%$ el fenómeno de la simplicidad medido desde las dimensiones teóricas iniciales establecidas en el instrumento (ver anexo 1).

La tabla 2, evidencia que, por extracción rotada, el componente 1 mantiene la mayor carga de varianza explicada con un poco más del $36 \%$, además, que al observar la matriz de componentes (Anexo 3), logra evidenciarse como el efecto de rotación Varimax logra crear grupos homogéneos saturados, lo que nos permite una mejor aproximación a la propuesta de nuevos constructos o ratificación de los ya existentes. A continuación, se muestra la síntesis obtenida por la extracción rotada.

La matriz de componentes rotados ha permitido resumir los 25 factores asociados a seis categorías empíricas que están aproximando la descripción de la simplicidad al interior de las organizaciones, estas categorías son:
Conciencia de su Trabajo (CT): esta dimensión fue planteada dentro del componente teórico inicial, el modelo la ratifica como la dimensión que tiene el mayor peso de la varianza explicada. Dentro de esta dimensión se destaca el factor EA 1.2 "Recibir incentivos por parte de la empresa para cooperar con sus compañeros de trabajo”. Al parecer, el incentivo está jugando un papel clave como estrategia para estimular la conciencia de trabajo por parte de los empleados.

Competencias específicas para el trabajo (CET): esta dimensión es una nueva recategorización que se obtiene al rotar los primeros seis componentes extraídos. Se define esta dimensión como las capacidades del saber hacer por parte de cada empleado, las cuales se encuentran embebidas dentro de su puesto de trabajo específicamente y desde donde se espera un desempeño específico. Emergen como características dos factores con carga factorial significativa, ellos son el AC 1.1. "La empresa la informó con claridad para que fue contratado"; y el factor CO 1.4 "La empresa ofrece espacios de trabajo acondicionados

Tabla 3.

Síntesis de la matriz rotada por Varimax y re -categorización dimensiones

\begin{tabular}{|c|c|c|c|}
\hline COMPONENTE & $\begin{array}{l}\text { DIMENSIÓNES RATIFICADAS U } \\
\text { RECATEGORIZADAS }\end{array}$ & FACTORES & $\begin{array}{c}\text { CARGA } \\
\text { FACTORIAL }\end{array}$ \\
\hline 1 & $\begin{array}{l}\text { Conciencia de su Trabajo (CT) } \\
\text { Varianza explicada del } 36,7 \%\end{array}$ & $\begin{array}{l}\text { EA 1.2 } \\
\text { AC } 1.4 \\
\text { EA } 1.3 \\
\text { CT } 1.3 \\
\text { AC } 1.5 \\
\text { CT } 1.4 \\
\text { CT } 1.5 \\
\text { CT } 1.1 \\
\text { EA } 1.1\end{array}$ & $\begin{array}{l}0,802 \\
0,683 \\
0,675 \\
0,637 \\
0,576 \\
0,554 \\
0,548 \\
0,518 \\
0,450\end{array}$ \\
\hline 2 & $\begin{array}{l}\text { Competencias Específicas para el } \\
\text { Trabajo (CET) Varianza explicada } \\
8,49 \% \text {. }\end{array}$ & $\begin{array}{l}\text { AC } 1.1 \\
\text { CO } 1.4 \\
C O 1.3 \\
A C 1.3 \\
A C 1.2\end{array}$ & $\begin{array}{l}0,786 \\
0,742 \\
0,661 \\
0,596 \\
0,576\end{array}$ \\
\hline 3 & $\begin{array}{l}\text { Capacitación (CAP) Varianza } \\
\text { explicada } 7,2 \%\end{array}$ & $\begin{array}{l}\text { HS } 1.4 \\
\text { HS } 1.5 \\
\text { CT } 1.2\end{array}$ & $\begin{array}{l}0,848 \\
0,790 \\
0,436\end{array}$ \\
\hline 4 & $\begin{array}{l}\text { Habilidad de Síntesis e Innovación } \\
\text { (HSI) Varianza explicada 5,7\% }\end{array}$ & $\begin{array}{l}\text { HS } 1.2 \\
\text { HS } 1.1 \\
\text { HS } 1.3\end{array}$ & $\begin{array}{l}0,791 \\
0,783 \\
0,707\end{array}$ \\
\hline 5 & $\begin{array}{l}\text { Autonomía y Bienestar (AUB) } \\
\text { Varianza explicada } 4,4 \%\end{array}$ & $\begin{array}{l}\text { EA } 1.4 \\
\text { CO } 1.5 \\
\text { CO } 1.2\end{array}$ & $\begin{array}{l}0,702 \\
0,590 \\
0,519\end{array}$ \\
\hline 6 & $\begin{array}{l}\text { Libertad de Acción (LBA) Varianza } \\
\text { explicada 4,10\% }\end{array}$ & $\begin{array}{l}\text { CO } 1.1 \\
\text { EA } 1.5\end{array}$ & $\begin{array}{l}0,786 \\
0,622\end{array}$ \\
\hline
\end{tabular}

Fuente: Calculo de los autores a través de la matriz de componentes rotados (Anexo3). 
para desarrollar sus actividades de forma cómoda y segura”. Se infiere que tanto el saber para que se es contratado como el tener un lugar físico apropiado aportan al desarrollo de una cultura de simplicidad dentro de las organizaciones del sector petroquímico. Se asocia a la ausencia de confusión como una característica propia de ambientes diseñados para actuar por simplicidad.

Capacitación (CAP): esta dimensión emerge como nueva recategorización, se obtiene como resultado de la rotación varimax de los primeros seis componentes. Puede comprenderse como todas aquellas acciones que las empresas realizan para mejorar las competencias de síntesis de sus empleados en búsqueda de obtener un mayor y mejor desempeño desde su capital humano. Destacan en esta dimensión dos factores ellos son: el HS 1.4 "La empresa lo capacitó en un método apropiado para resolver problemas", y el segundo es HS 1.5 "Preparación en la empresa para desarrollar capacidad de síntesis frente a los problemas". Se infiere que se refiere a un tipo específico de capacitación y no una general.

Habilidad de Síntesis e Innovación (HSI): esta dimensión es complementada con la característica de Innovación. Teóricamente se planteó solo como la habilidad de síntesis, sin embargo, la prueba empírica efectuada el modelo factorial rotado, agrupo el factor de innovación asociándolo a la capacidad de síntesis. Es una dimensión compuesta por tres factores todos con carga factorial significativa, ellos son: HS 1.2 "Presentación de informes de trabajo concretos y precisos"; HS 1.1 "Entrega de información precisa a los clientes y compañeros de trabajo"; HS 1.3 "Usa la iniciativa, creatividad e ingenio para resolver problemas de la empresa”. La dimensión de habilidad de síntesis se infiere asociada entonces a los factores de simplicidad. La habilidad de síntesis implica las competencias del empleado para pensar críticamente, argumentar y precisar.

Autonomía y Bienestar (AUB): Esta dimensión está asociada a las categorías teóricas Comodidad y Espiritu de ayuda. Autonomía y Bienestar implican que el desarrollo de la simplicidad requiere de brin- dar al empleado aspectos de comodidad en su trabajo desde donde pueda sentirse en disposición para ayudar a otros, pero bajo los rasgos propios del uso de su propia voluntad. El factor predominante que emerge de la extracción por rotación, es el factor EA 1.4 "La capacidad de resolver problemas sin que medie tanto trámite administrativo”.

Libertad de Acción (LBA): esta dimensión se encuentra asociada a las dimensiones teóricas de Comodidad y Espiritu de ayuda. Se comprende como el nivel de empoderamiento que el empleado ha recibido para actuar con libertad, a conciencia y de comunicar lo que piensa sin temores. Está compuesta por dos factores, el de mayor carga factorial es el CO 1.1 "La empresa le da libertad para personalizar su espacio físico de trabajo". Se infiere que la libertad de acción es una consecuencia de cómo percibe el empleado los factores de comodidad que brinda la empresa que pueden ser traducidos en conductas de cooperación y ayuda a los demás.

En resumen, el campo de fuerzas para desarrollar una cultura de simplicidad en empresas del sector petroquímico de la ciudad de Cartagena de indias, puede sintetizarse en la Figura 1.

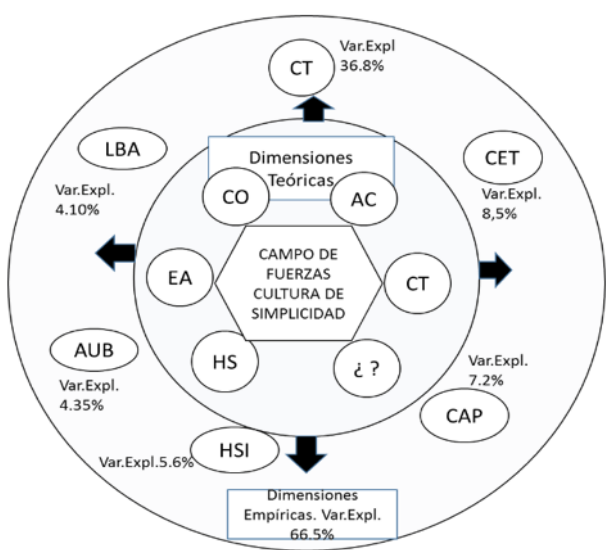

Figura 1. Campo de Fuerzas alrededor de una cultura de simplicidad en las empresas del sector petroquímico de Cartagena de Indias. Fuente: Elaboración propia de los autores con base en el análisis de datos y Modelo factorial.

El campo de fuerzas se configura en primer lugar, por el contenido teórico expresado en cinco 
dimensiones (primer círculo interno) en el que se especificaron las dimensiones Comodidad (CO); Ausencia de confusión (AC); Conciencia del Trabajo (CT); Habilidad de Síntesis (HS); y Espiritu de Ayuda (EA). La evidencia empírica obtenida por el modelo factorial con extracción basada en rotación varimax, permite obtener las dimensiones empíricas que explican un poco más del $66 \%$ la varianza. Estas dimensiones empíricas que constituyen el campo de fuerzas (circulo mayor), son en su orden por Explicación de Varianza las siguientes: Conciencia en el Trabajo (CT); Competencias específicas para el trabajo (CET); Capacitación (CAP); Habilidades de síntesis e Innovación (HSI); Autonomía y Bienestar (AUB); Libertad de acción (LBA).

\section{Conclusiones}

Los resultados de la investigación adelantada sobre el campo de fuerzas alrededor de una cultura de simplicidad como estrategia para incorporar en los modelos de gestión con el propósito de mejorar el desempeño de la organización, ha permitido explorar y obtener hallazgos de interés, que, aunque no se pretende exponerlos para la generalización universal, si es pertinente hacer la generalización analítica que corresponde al caso.

El primer hallazgo del que puede dar cuenta la investigación realizada en empresas del sector petroquímico, es, que emergen de manera exploratoria seis dimensiones que pueden ayudar a comprender la simplicidad como estrategia para ser incorporada en la gestión de las organizaciones, estas dimensiones son en su orden por explicación de varianza: Conciencia de su trabajo; Competencias específicas para el trabajo; Capacitación; Habilidad de síntesis e Innovación; Autonomía y Bienestar; Libertad de Acción.

El segundo hallazgo se establece desde la evidencia empírica, la dimensión "Conciencia de su trabajo” presenta la mayor carga de varianza explicada con un poco más del $36 \%$, y dentro de esta dimensión el factor Incentivo recibido, presenta la mayor carga factorial. Lo que se evidencia, es que la simplicidad antes que ser un asunto de lógicas computacionales, es primero un asunto humano que se desenvuelve por lógicas conectivas, de comprensión, cognición, y percepción, que infiere la necesidad psicológica del individuo de sentirse reconocido, premiado.

Un tercer hallazgo es la evidencia empírica acerca de la importancia para desarrollar una estrategia de simplicidad, de que cada trabajador tenga claridad consiente de para qué fue contratado. Esto queda claro en la dimensión "Competencias específicas para el Trabajo", en que su factor de mayor carga factorial es la claridad que sobre su puesto de trabajo tiene el trabajador. Esta hace menos complejo su desempeño.

Un cuarto hallazgo se evidencia en la dimensión “Capacitación”, en ella se encuentra el factor que muestra la importancia de que la empresa capacite a sus empleados en métodos para resolver problemas y el desarrollo de capacidad de síntesis. La síntesis bajo la mirada del desarrollo de capacidad crítica y de comunicación asertiva para expresar de manera apropiada lo que se piensa frente al fenómeno o problema observado.

Un quinto hallazgo es encontrado a través de la dimensión "Habilidad de síntesis e Innovación”, en el que se evidencia tres factores de carga factorial significativa y que se comprometen con la capacidad del trabajador para preparar informes concretos y precisos, con la capacidad de entregar información a clientes y compañeros, y la capacidad para usar la creatividad, el ingenio frente a los problemas. Esta dimensión se planteó inicialmente de forma teórica como habilidad de síntesis, sin embargo, la evidencia empírica la asocia factorialmente a la innovación.

Un sexto hallazgo se obtiene desde la dimensión de Autonomía y Bienestar, en el que el factor de mayor carga factorial indica que para el desarrollo de una cultura de simplicidad es necesario considerar la reducción de tanto trámite administrativo y que esta reducción pueda ser orientada al empoderamiento de los trabajadores para tomar decisiones y resolver problemas con mayor autonomía. 
Un séptimo hallazgo se evidencia en la dimensión Libertad de Acción, la implicación que para la cultura de simplicidad tiene el alcance de la libertad que tenga el trabajador para personalizar su puesto de trabajo o sentirse libre de presiones indebidas, además de que ésta le permita actuar de forma espontánea cooperando con sus compañeros de trabajo.

De otra parte, tal como se muestra en la figura 1, se pudo establecer conceptualmente el modelo de las fuerzas que están alrededor de una cultura de simplicidad.

En cuanto a nuevas investigaciones sobre este tema, es posibleabordarlaestructuración demodelos de causalidad dado los primeros hallazgos obtenidos por la aplicación del modelo factorial confirmatoria con base en rotación varimax. Las cargas factoriales y los porcentajes de explicación de varianza dan cuenta de las variables en su orden de importancia. La causalidad es relevante investigarla toda vez que se pueden encontrar recomendaciones que permitan mejorar los desempeños de las empresas basados en premisas de simplicidad organizacional.

Por otra parte, la evidencia obtenida por el modelo factorial es clave para diseñar instrumentos de medición de simplicidad en las organizaciones al menos del sector petroquímico, además de haberse validado el instrumento que permite mejorar los análisis y diagnósticos en esta materia. Es indudable que esta investigación no agota el tema ni pretende eso, es en primer lugar una investigación exploratoria que arroja resultados de interés para diseñar investigaciones futuras, que pueden ser abordas por técnicas de ecuaciones estructurales o modelos lineales. Sin embargo, es recomendable que el estudio de la simplicidad en la perspectiva de las organizaciones pueda ser complementado con investigación cualitativa a través de observación participante y entrevistas en profundidad, y de aquí podría derivarse un enfoque metodológico mixto, con características cualitativas-cuantitativas.

\section{Referencias}

Argyris, C. (1997). Theories of action, double-loop learning in organizational learning. Brighton de Boston, Massachusetts, Estados Unidos: Harvard Business Review.

Ashkenas, R. (2007). Simplicity. Minded Management. https://hbr. org/2007/12/simplicity-minded-management

Balmes, J. (1860). Filosofía Fundamental. Barcelona, España: Imprenta del diario de Barcelona.

Cronbach, L. J. (1951). Coefficient alpha and the internal structure of tests. Psychometrika. 16, 297-334.

De Bono, E. (1998). Simplicidad/técnicas de pensamiento para librarse de la tiranía de la complejidad. Publicado originalmente en ingles por The Penguin Group. Barcelona, España: Ediciones Paidós.

Di Tore, P. A., Di Tore, S., Mangione, G. R., \& Corona, F. (2014). El espacio empático en la educación: representación del espacio y empatía, de Piaget a la didáctica de la "simplicidad". Reladei, 3(2), 95-113.

Gore, E. (2012). El Próximo Management: Acción, practica y aprendizaje. Barcelona, España: Editorial Management.

Grant, R. M. (1996). Toward a Knowledge-based theory of the firm. Strategic Management Journal, 17, 109-122.

Hoffman, M. L. (1987). La aportación de la empatía a la justicia y al juicio moral. En N. Eisemberg \& J. Strayer (Eds.), La empatía y su desarrollo (pp. 59-93). Bilbao, España: Desclée de Brouwer.

Krippendorff, K. (1990). Metodología de análisis de contenido: Teoría y práctica. Primera Edición. Barcelona, España: Editorial Paidós.

Lewin, K. (1951). Field Theory in Social Scienc. New York, Estados Unidos: Harper \& Row.

Morin, E. (1994). Introducción al pensamiento complejo. Primera edición. Barcelona, España: Gedisa.

Nisbett, R. E. (2016). Minware : Herramientas para pensar mejor. 
México: Editorial Debate.

Katsuhiko, O. (1987). Dinámica de Sistemas. México: Prentice -

Hall, Hispanoamericana.

Perrow, C. (1986). Complex Organizations: A Critical Essay. Nueva York, Estados Unidos: McGraw-Hill Higher Education.

Robledo, J.; Del Rio, J.; Martínez, O; \& Ruiz, G. (2015). La Gestión del Conocimiento Organizacional: Fundamentos Teóricos. Baja California., México: Editorial Jorales \& Editorial Universidad Autónoma de Baja California.

Robledo, J. \& Ruiz, C. (2017). Campo de Fuerzas Alrededor de una Cultura Organizacional Caracterizada por la Simplicidad: Caso Agencias de Viajes (Tesis de Maestría). Universidad Tecnológica de Bolívar, Cartagena, Colombia.

Robledo, J. \& Santamaría, A. (2016). Análisis del Campo de Fuerzas de la cultura de simplicidad y la empatía en las organizaciones”. En IV Congreso Internacional de la Red de Posgrados de Investigación Latinos en Administración y Estudios Organizacionales (Red Pilares) VII parte, pág. 1765: complementariedades y contradicciones entre administración y estudios organizacionales.

Sand, G., (2010). Historia de George Sand. https:// historiaenfemenino.wordpress.com/2010/12/16/george-sand/

Segall, K. (2012). Entrevista a Ken Segall, Consultor creativo que ha trabajado para Apple, IBMy Dell. Revista HarvardDeusto, (112), $20-24$.

Teece, D. J., Pisano, G. \& Shuen, A. (1997). Dynamic capabilities and strategic Management”. Strategic Management Journal, 18(7), 509-533.

Tomasko, R. (1993). Repensar la Empresa: La arquitectura del cambio. Primera edición, Barcelona, España: Ediciones Paidós.

Wernerfelt, B. (1984). A Resource-based view of the firm”. Strategic Management Journal, 5, 171-180. 
Anexo 1.

Instrumento aplicado

\begin{tabular}{|c|c|c|c|c|c|}
\hline ÍTEMS & 1. & 2. & 3. & 4. & 5. \\
\hline 1.1 La empresa le da libertad para personalizar su e & & & & & \\
\hline 1.2 La empresa le brinda espacios físicos para su bienestar personal & & & & & \\
\hline $\begin{array}{l}\text { 1.3 La empresa le ofrece las herramientas o elementos necesarios para realizar } \\
\text { su trabajo con eficiencia y comodidad. }\end{array}$ & & & & & \\
\hline $\begin{array}{l}\text { 1.4 La Empresa le ofrece espacios de trabajo acondicionados para desarrollar } \\
\text { sus actividades de forma cómoda y segura. }\end{array}$ & & & & & \\
\hline $\begin{array}{l}\text { 1.5 La Empresa le ofrece un canal de comunicación apropiado para informar } \\
\text { sus incomodidades en el trabajo. }\end{array}$ & & & & & \\
\hline 2.1 La empresa le informó con claridad para que fue contratado & & & & & \\
\hline $\begin{array}{l}2.2 \text { La empresa le dio una inducción clara y suficiente para el desempeño de } \\
\text { sus funciones }\end{array}$ & & & & & \\
\hline $\begin{array}{l}2.3 \text { Las competencias por las cuales usted fue contratado corresponden a las } \\
\text { funciones asignadas en su trabajo. }\end{array}$ & & & & & \\
\hline $\begin{array}{l}2.4 \mathrm{La} \text { empresa le ha brindado regularmente acompañamiento en su puesto de } \\
\text { trabajo para el desempeño de sus funciones. }\end{array}$ & & & & & \\
\hline $\begin{array}{l}2.5 \text { La empresa se ha preocupado por su proceso de crecimiento en } \\
\text { aprendizaje y nuevo conocimiento. }\end{array}$ & & & & & \\
\hline $\begin{array}{l}\text { 3.1 La empresa le ha brindado un conocimiento en el cual usted sabe cómo } \\
\text { se vincula su trabajo con el de los demás. }\end{array}$ & & & & & \\
\hline $\begin{array}{l}\text { 3.2 La empresa le ha brindado conocimiento acerca de cómo se benefician } \\
\text { los clientes con su trabajo. }\end{array}$ & & & & & \\
\hline $\begin{array}{l}\text { 3.3 La empresa le ha brindado un conocimiento desde donde usted puede } \\
\text { saber que competencias son las claves de su trabajo }\end{array}$ & & & & & \\
\hline $\begin{array}{l}\text { 3.4 La empresa le ha brindado acompañamiento para que usted conozca mejor } \\
\text { su trabajo. }\end{array}$ & & & & & \\
\hline $\begin{array}{l}\text { 3.5 La valoración del desempeño que le hace la empresa es justa y coherente } \\
\text { con las competencias por las que lo contrataron. }\end{array}$ & & & & & \\
\hline $\begin{array}{l}\text { 4.1 Usted siempre entrega información precisa a sus clientes o compañeros } \\
\text { de trabajo. }\end{array}$ & & & & & \\
\hline 4.2 Usted siempre presenta informes de trabajo cortos y precisos & & & & & \\
\hline $\begin{array}{l}\text { 4.3 Usted siempre hace uso de su creatividad, iniciativa e ingenio para } \\
\text { resolver problemas de la empresa }\end{array}$ & & & & & \\
\hline 4.4 La empresa lo capacito en un método apropiado para resolver problemas & & & & & \\
\hline $\begin{array}{l}4.5 \text { Usted ha sido preparado en la empresa para desarrollar una excelente } \\
\text { capacidad de síntesis frente a los problemas. }\end{array}$ & & & & & \\
\hline 5.1 La empresa le ha facilitado la cooperación con sus compañeros de trabajo. & & & & & \\
\hline $\begin{array}{l}\text { 5.2 Usted ha recibido incentivos de la empresa para cooperar con sus } \\
\text { compañeros de trabajo. }\end{array}$ & & & & & \\
\hline 5.3 La empresa ha creado un entorno de cooperación & & & & & \\
\hline 5.4 Usted puede resolver problemas sin tanto trámite administrativo. & & & & & \\
\hline 5.5 La empresa le permite dar avuda a otros de forma autónoma & & & & & \\
\hline
\end{tabular}

Fuente: Diseño por los autores con base marco teórico. Alpha de Cronbach =0,9. 1: totalmente en desacuerdo, 2: en desacuerdo, 3: Ni en desacuerdo ni en acuerdo, 4: de acuerdo, 5: Totalmente de acuerdo Del ítem 1.1 al 1.5 la dimensión teórica comodidad (CO). Del ítem 2.1 al 2.5 la dimensión teórica ausencia de confusión (AC) en el trabajo. Del ítem 3.1 al 3.5 la dimensión teórica Conciencia de su trabajo (CT). Del ítem 4.1 al 4.5 la dimensión teórica Habilidades de Síntesis (HS). Del ítem 5.1 al 5.5 la dimensión teórica espíritu de ayuda (EA) 
Anexo 2.

Matriz de Componentes principales por extracción sin rotación

\begin{tabular}{|c|c|c|c|c|c|c|}
\hline \multicolumn{7}{|c|}{ Matriz de componente ${ }^{3}$} \\
\hline & \multicolumn{6}{|c|}{ Componente } \\
\hline & 1 & 2 & 3 & 4 & 5 & 6 \\
\hline $\begin{array}{c}\text { AC1.5: La empresa se ha preocupado por su proceso de crecimiento } \\
\text { en aprendizaje y nuevo conocimiento }\end{array}$ & .793 & -.086 & .043 & -.184 & .148 & 208 \\
\hline $\begin{array}{l}\text { CT1.1: La emprersa le ha brindado un conocimiento en el cuál usted } \\
\text { sabe como se vincula su trabajo con el de los demás }\end{array}$ & .784 & -.179 & -.119 &,- 051 & .048 &,- 069 \\
\hline $\begin{array}{l}\text { CT1.3: La Empresa le ha brindado un conocimiento desde donde } \\
\text { usted puede saber que competencias son clave de su trabajo }\end{array}$ & .771 & -.276 & -.219 & .064 & -.066 & .019 \\
\hline $\begin{array}{l}\text { CT1.4. La empresa le ha brindado acompańamiento para que usted } \\
\text { conozca mejor su trabajo }\end{array}$ & .763 & -.225 & -.167 &,- 147 & .020 & .005 \\
\hline $\begin{array}{l}\text { CT1.2 La empresa le ha brindado conocimiento acerca de como se } \\
\text { benefician los clientes son su trabajo }\end{array}$ & .737 & .006 & -.034 & .211 & .105 &,- 161 \\
\hline $\begin{array}{l}\text { EA1.1: La Empresa le ha facilitado la cooperación con sus } \\
\text { compañeros de trabajo }\end{array}$ & .724 & 097 & -.146 & .004 &,- 005 & .075 \\
\hline $\begin{array}{c}\text { AC1.4. La empresa le ha brindado regularmente acompañamiento en } \\
\text { su puesto de trabajo para el desempeño de sus funciones }\end{array}$ & .717 & -.207 & .129 & -137 & .173 & .400 \\
\hline EA1 .3: La empresa ha creado un entorno de coopearación & .700 & -.173 & -.302 & 191 &,- 008 & .128 \\
\hline $\begin{array}{l}\text { AC1.2: La Empresa le cio una inducción clara y suficiente para el } \\
\text { desempeño de sus funciones }\end{array}$ & 681 & .112 & .066 &,- 197 &,- 323 &,- 105 \\
\hline $\begin{array}{l}\text { HS1,5: Usted ha sido preparado en la empresa para desarrollar } \\
\text { capacidad de sintesis frente a los problemas }\end{array}$ & .658 & .013 & -.418 &,- 190 & ,085 &,- 317 \\
\hline $\begin{array}{l}\text { C01.5: La Empresa le ofrece un canal de comunicación apropiado } \\
\text { para informar sus incomodidades en el trabajo }\end{array}$ & .639 & 149 & .356 &,- 365 & .047 & .125 \\
\hline $\begin{array}{l}\text { HS1.4: La empresa lo capacitó en un método apropiado para resolver } \\
\text { problemas }\end{array}$ & .639 & .027 & -.404 &,- 200 & .082 & -.423 \\
\hline AC1.1: La empresa le informó con claridad para que fue contratado & .616 & -.063 & 351 & .074 & -.400 &.,- 222 \\
\hline $\begin{array}{l}\text { C01.3: La empresa le ofrece las herramientas necesarias para } \\
\text { realizar su trabajo con eficiencia y comodidad }\end{array}$ & 607 & -.091 & .501 & -.161 & -.009 & .071 \\
\hline $\begin{array}{c}\text { C01.2: La empresa le brinda espacios físic os para su bienestar } \\
\text { personal }\end{array}$ & .591 & .304 & .176 &,- 078 & .284 &,- 233 \\
\hline $\begin{array}{l}\text { AC1.3: Las competencias por las cuales usted fue contratado } \\
\text { comesponden a las funciones asignadas en su trabajo }\end{array}$ & .575 &,- 193 & .311 & .213 &,- 142 &,- 062 \\
\hline $\begin{array}{l}\text { CO 1.4. La empresa le ofrece espacios de trabajo acondicioandos } \\
\text { para desarrollar sus activiclades de forma comoda y segura }\end{array}$ & .564 & -.039 & .488 &,- 008 & -.242 & .010 \\
\hline $\begin{array}{c}\text { CT1.5: La valoración del desempefí que le hace la empresa es justa } \\
\text { y coherente con las competencias por las que lo contrataron }\end{array}$ & .544 & -.304 &,- 088 & .479 & -.140 &,- 009 \\
\hline $\begin{array}{c}\text { EA1.2: Usted ha recibido incentivos de la empresa para cooperar con } \\
\text { sus compańeros de trabajo }\end{array}$ & .488 & -.325 & -.267 & .230 & .203 & .381 \\
\hline HS1.2: Usted siempre presenta infomes de trabajo cortos y precisos & .271 & .708 & -.225 & 081 & -.092 & 209 \\
\hline $\begin{array}{c}\text { HS1.1: Usted siempre entrega información precisa a sus clientes o } \\
\text { compañeros de trabajo }\end{array}$ & .432 & .549 &,- 303 & .056 &,- 395 & 030 \\
\hline $\begin{array}{c}\text { HS1.3: Usted siempre hace uso de su creatividad, iniciativa e ingenio } \\
\text { para resolver problemas de la empresa }\end{array}$ & .334 & .539 & -.068 & .130 & -.204 & 290 \\
\hline Espinitu de Ayuda1.4 & .402 & .458 & .164 &,- 215 & .418 &,- 030 \\
\hline $\begin{array}{c}\text { CO 1.1: La empresa le da libertad para personalizar su espacio físico } \\
\text { de trabajo }\end{array}$ & 211 & .117 & .312 & .589 & .253 &,- 276 \\
\hline EA 1.5: La empresa le permite dar ayuda a otros de forma autonoma & 340 & .425 & .138 & .454 & .268 & .040 \\
\hline
\end{tabular}

Mètodo de extracción: análisis de componentes principales.

a. 6 com ponentes extraídos.

Fuente: Los autores con base en los datos obtenidos y utilizando el SPSS.

CO: Dimensión de Comodidad

AC: Dimensión Ausencia de Confusión

CT: Dimensión Conciencia de su trabajo

HS: Dimensión Habilidades de Síntesis

EA: Espíritu de Ayuda 
Anexo 3.

Matriz de componentes rotados confirmatorio por método Varimax

\begin{tabular}{|c|c|c|c|c|c|c|}
\hline \multicolumn{7}{|c|}{ Matriz de componente rotado ${ }^{2}$} \\
\hline & \multicolumn{6}{|c|}{ Componente } \\
\hline & 1 & 2 & 3 & 4 & 5 & 6 \\
\hline $\begin{array}{l}\text { EA1.2: Usted ha recibido incentios de la empresa para cooperar } \\
\text { con sus compañeros de trabajo }\end{array}$ & .802 &,- 031 & .065 & .014 & .010 & .078 \\
\hline $\begin{array}{l}\text { AC1 .4: La empresa le ha brindado regulamente ac ompañamiento } \\
\text { en su puesto de trabajo para el desempeño de sus funciones }\end{array}$ & 683 & .365 & .050 & .022 & .418 & -.067 \\
\hline EA1.3: La empresa ha creado un entomo de cooperación & .675 & .176 & .359 & .190 & -.041 & .106 \\
\hline $\begin{array}{l}\text { CT1.3: La empresa le ha brindado un conocimiento desde donde } \\
\text { usted puede saber que competencias son la clave de su trabajo }\end{array}$ & .637 & .332 & .453 & .075 &,- 021 & .030 \\
\hline $\begin{array}{l}\text { AC1.5: La empresa se ha preocupado por su proceso de } \\
\text { crecimiento en aprendizajey nuevo conocimiento }\end{array}$ & .576 & .368 & .270 & 101 & 431 &,- 036 \\
\hline $\begin{array}{l}\text { CT1.4: La empresa le ha brindado ac om pañamiento para que } \\
\text { usted conoz ca mejor su trabaio }\end{array}$ & .554 & .323 & .485 & ,031 & .170 &,- 075 \\
\hline $\begin{array}{l}\text { CT1 .5: La valoración del desempeńo que le hace la empres a es } \\
\text { justay coherente con las competencias por las que lo } \\
\text { contrataron }\end{array}$ & .548 & .316 & 196 & ,039 &,- 311 & .329 \\
\hline $\begin{array}{l}\text { CT1.1: La empres a le ha brindado un conocimiento en el cual } \\
\text { usted sabe cómo se vincula su trabajo con el de los demás. }\end{array}$ & .518 & .346 & .500 & .040 & 166 & .060 \\
\hline $\begin{array}{l}\text { EA1.1: La empresa le ha facilitado la cooperación con sus } \\
\text { compan̂eros de trabajo }\end{array}$ & .450 & .266 & .373 & .320 & 200 & .076 \\
\hline $\begin{array}{l}\text { AC1.1: La empresa le informó con claridad para que fue } \\
\text { contratado }\end{array}$ & .092 & .786 & .242 & 102 &,- 052 & 152 \\
\hline $\begin{array}{l}\text { CO 1.4: La empresa ofrece espacios de trabajo acondicionados } \\
\text { para desamollar sus actividades de forma comoda y segura }\end{array}$ & .152 & .742 & .016 & 071 & 166 & .103 \\
\hline $\begin{array}{l}\text { CO 1.3: La Empresa le ofrece herramientas nec es arias para } \\
\text { realizar su trabajo con eficiencia y comodidad }\end{array}$ & .238 & .661 & .033 & -.052 & .398 & .051 \\
\hline $\begin{array}{l}\text { AC1.3: Las competencias por las cuales usted fue contratado } \\
\text { corrersponden a las funciones asignadas en su trabajo }\end{array}$ & .309 & .596 & 110 & -.027 &,- 004 & 264 \\
\hline $\begin{array}{l}\text { AC1.2: La empresa le dio una inducción clara y suficiente para el } \\
\text { desempeño de sus funciones. }\end{array}$ & 151 & .576 & .404 & .299 & 136 & -.095 \\
\hline $\begin{array}{l}\text { HS1.4: La empres a lo capacitó en un método apropiado para } \\
\text { resolver problemas }\end{array}$ & .204 & .085 & .848 & .097 & .141 & .019 \\
\hline $\begin{array}{l}\text { HS1.5: Usted ha sido preparado en la empresa para des arollar } \\
\text { capacidad de síntesis frente a los problemas }\end{array}$ & .286 & .075 & .790 & .128 & 151 & -.010 \\
\hline $\begin{array}{l}\text { CT1.2: La empresa le ha brindado conocimiento acerca de como } \\
\text { se benefician los cliertes con su trabajo }\end{array}$ & .404 & .312 & .436 & .137 & .138 & ,369 \\
\hline $\begin{array}{l}\text { HS1.2. Usted siempre presenta informes de trabajo cortos y } \\
\text { precisos }\end{array}$ & .017 &,- 084 & .082 & .791 & .189 & ,093 \\
\hline $\begin{array}{l}\text { HS1.1: Usted siempre entrega información precisa a sus clientes } \\
\text { o compañeros de trabajo }\end{array}$ &, 040 & 153 & .316 & .783 &,- 054 & -.011 \\
\hline $\begin{array}{l}\text { HS1.3: Usted siempre hace uso de su creatividad, iniciativa e } \\
\text { ingenio para resolver problemas de la empresa }\end{array}$ & .117 & 121 &,- 037 & .707 & 118 & .077 \\
\hline $\begin{array}{l}\text { EA1.4: Usted puede resolver problemas sin tarto tramite } \\
\text { administrativo }\end{array}$ &,- 011 & .048 & 197 & .216 & .702 & 2000 \\
\hline $\begin{array}{l}\text { CO1.5: La empresa le ofrece un c anal de comunicación } \\
\text { apropiado para informar sus incomodidades en su trabajo }\end{array}$ & 185 & .528 & 134 & 149 & .590 &,- 087 \\
\hline $\begin{array}{l}\text { Co 1.2: La empresa le brinda espacios físicos para su bienestar } \\
\text { personal }\end{array}$ & ,055 & .260 & .383 & .146 & .519 & .325 \\
\hline $\begin{array}{l}\text { CO 1.1: La empresa le da libertad para personalizar su espacio } \\
\text { físico de trabajo }\end{array}$ & .008 & .153 & .002 &,- 036 & ,028 & .786 \\
\hline $\begin{array}{l}\text { EA1.5: La Empresa le permite dar ayuda a otros de forna } \\
\text { autonoma }\end{array}$ & 140 & .032 &,- 029 & .355 & .245 & .622 \\
\hline
\end{tabular}

Método de extracción: análisis de componentes principales.

a. La rotación ha convergido en 10 iteraciones.

Fuente: Los autores con base en los datos obtenidos y utilizando el SPSS.

CO: Dimensión de Comodidad

AC: Dimensión Ausencia de Confusión

CT: Dimensión Conciencia de su trabajo

HS: Dimensión Habilidades de Síntesis

EA: Espiritu de Ayuda 
Anexo 4.

Estadísticos de prueba de fiabilidad para el instrumento y el uso de la técnica factorial

1. Prueba de Fiabilidad y Consistencia interna del instrumento aplicado.

\begin{tabular}{|l|c|}
\hline \multicolumn{1}{|c|}{ Dimensión } & $\begin{array}{c}\text { Alpha } \\
\text { Cronbach }\end{array}$ \\
\hline Comodidad (CO) & 0,704 \\
\hline Ausencia Confusión (AC) & 0,817 \\
\hline $\begin{array}{l}\text { Conciencia de su trabajo } \\
\text { (CT) }\end{array}$ & 0,862 \\
\hline $\begin{array}{l}\text { Habilidad de Síntesis } \\
\text { (HS) }\end{array}$ & 0,699 \\
\hline Espiritu de ayuda (EA) & 0,655 \\
\hline
\end{tabular}

\begin{tabular}{|l|l|}
\hline Alpha Cronbach Global & 0,921 \\
\hline
\end{tabular}

Fuente: Cálculos con SPSS.

2. Pruebas para verificación en la viabilidad de aplicar modelo factorial de primeros componentes.

Prueba de KMO y Bartlett para extracción no rotada

\begin{tabular}{|llr|}
\hline $\begin{array}{l}\text { Medida Kaiser-Meyer-Olkin de adecuación de } \\
\text { muestreo }\end{array}$ &, 861 \\
Prueba de esfericidad & Aprox. Chi-cuadrado & 1603,861 \\
de Bartlett & gl & 300 \\
& Sig. &, 000 \\
\hline
\end{tabular}

Fuente: Cálculos con SPSS.

Prueba de KMO y Bartlett para extracción rotada con Varimax

\begin{tabular}{|llr|}
\hline $\begin{array}{l}\text { Medida Kaiser-Meyer-Olkin de adecuación de } \\
\text { muestreo }\end{array}$ &, 861 \\
Prueba de esfericidad & Aprox. Chi-cuadrado & 1603,861 \\
de Bartlett & gl & 300 \\
& Sig. &, 000 \\
\hline
\end{tabular}

Fuente: Cálculos con SPSS. 